\title{
Birnesitas obtenidas mediante cambio iónico. Evolución estructural con la calcinación
}

\author{
V. RIVES*, M. DEL ARCO, O. PRIETO \\ Departamento de Química Inorgánica, Universidad de Salamanca, Salamanca, España.
}

\begin{abstract}
Se han preparado birnesitas con $\mathrm{Na}^{+} \mathrm{o} \mathrm{K}^{+}$en la interlámina mediante un método sol-gel, con una relación inicial glucosa/catión de 1 y 1.5. Estas muestras se han sometido a cambio iónico con los cationes $\mathrm{Li}^{+}, \mathrm{Mg}^{2+} \mathrm{y} \mathrm{Cu}^{2}$, caracterizando las muestras intercambiadas por diversas técnicas (análisis químico elemental, difracción de rayos X, espectroscopía FT-IR, análisis térmico y determinación de superficie específica). En ningún caso se alcanza un cambio iónico completo de los iones sodio o potasio iniciales, aunque el sodio se intercambia en mayor medida que el potasio. El intercambio es más efectivo al incorporar el $\mathrm{Li}^{+}$, menor en el caso del $\mathrm{Cu}^{2+} \mathrm{y}$ menos eficiente, todavía, al incorporar $\mathrm{Mg}^{2+}$. El cambio iónico se produce topotácticamente, manteniéndose en todos los casos la estructura laminar con una distancia interlaminar de, aproximadamente, $7 \AA$ Å. Las muestras son estables hasta $600^{\circ} \mathrm{C}$; a partir de esta temperatura se produce la transformación a estructura túnel $2 \times 2$ (criptomelano) en el caso de la birnesita de $\mathrm{K}$ y a la espinela de $\mathrm{Li}-\mathrm{Mn}\left(\mathrm{LiMn}_{2} \mathrm{O}_{4}\right)$ en el caso de tener Li en la interlámina.
\end{abstract}

Palabras claves: birnesita, óxidos de Mn, no estequiometría.

\section{Birnessites prepared by ion exchange. Structural evolution with temperature}

Birnessites containing $\mathrm{Na}^{+}$or $\mathrm{K}^{+}$in the interlayer have been prepared following a sol-gel method, with an initial glucose/cation ratio of 1 or 1.5. The solids have been submitted to ion exchange with $\mathrm{Li}^{+}, \mathrm{Mg}^{2+}$, and $\mathrm{Cu}^{2+}$, and all the materials have been characterised by several physicochemical techniques (elemental chemical analysis, X-ray diffraction, FT-IR spectroscopy, thermal analysis and specific surface area assessment). Total cation exchange was not achieved in any case, although sodium is better exchanged than potassium. Exchange is larger for $\mathrm{Li}^{+}$, lower for $\mathrm{Cu}^{2+}$ and even lower for $\mathrm{Mg}^{2+}$. Ionic exchange is topotactic, and the layered structure is maintained in all cases, with an interlayer spacing close to $7 \AA$. The samples are stable up to $600{ }^{\circ} \mathrm{C}$; above this temperature cryptomelane (a $2 \times 2$ tunnel structure) is formed in most of the samples containing potassium, while $\mathrm{LiMn}_{2} \mathrm{O}_{4}$ spinel is formed is $\mathrm{Li}$ was present in the birnessite.

Keywords: birnessite, Mn oxides, non-stoichiometry.

\section{INTRODUCCIÓN}

Las birnesitas son óxidos de manganeso laminares, formados por láminas de octaedros $\left[\mathrm{MnO}_{6}\right]$ que comparten aristas, entre las cuales se sitúan cationes, normalmente alcalinos o alcalino-térreos, junto a moléculas de agua, para compensar el exceso de carga negativa producido por las láminas octaédricas de Mn (Figura 1) [1-11]. La fórmula estructural se puede representar como $\mathrm{A}_{x} \mathrm{MnO}_{2 \pm \mathrm{y}} \mathrm{z}\left(\mathrm{H}_{2} \mathrm{O}\right)$ donde A representa el catión interlaminar; el estado de oxidación del manganeso se encuentra entre 3.6 y 3.8. El espaciado típico es aproximado a $7 \AA$ A pero tras hidratación el espaciado aumenta hasta $10 \AA$ (buserita). También se ha obtenido e identificado una forma deshidratada con espaciado laminar de aproximadamente 5.5 Å. Estos compuestos presentan capacidad de cambio iónico que, en algunas ocasiones, conlleva una variación del espaciado laminar.

Uno de los aspectos importantes de los óxidos de manganeso laminares es su capacidad para formar compuestos con estructura túnel y espinelas, tras un tratamiento térmico adecuado. Las estructuras túnel de tamaño y forma conocidas son ransdelita (2x1), holandita (2x2), romanechita (2x3), todorokita (3x3) y una sintética $\mathrm{Rb}_{0.27} \mathrm{MnO}_{2}(2 \times 5)$ [12], así como un óxido de manganeso con cationes alcalinos $(2 \times 4)$ designado RUB-7 [13], y otro con composición estructural de holandita y pirolusita, con túneles $(2 \times 2)$ y (2x3) [14]. Dentro de la familia de las holanditas el túnel puede estar ocupado por otros cationes formando

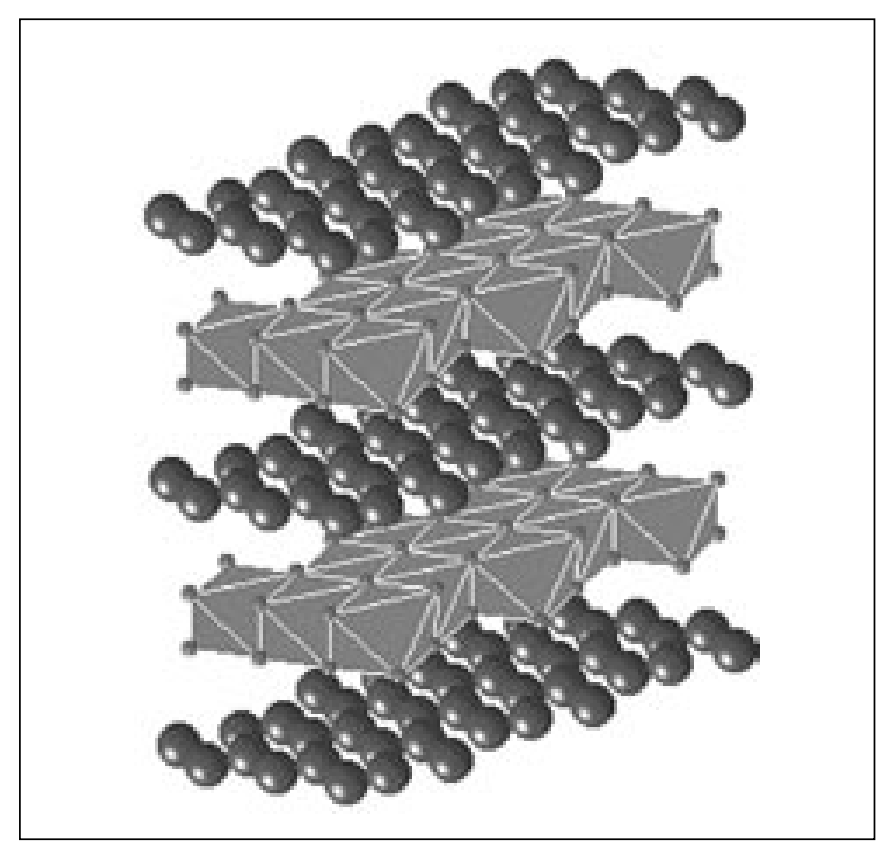

Figura 1.-Modelo de estructura de la birnesita 
así una serie isomorfa: $\mathrm{Ba}^{2+}$ (holandita), $\mathrm{Pb}^{2+}$ (coronadita), $\mathrm{Na}^{+}$(manjiroita) y $\mathrm{K}^{+}$(criptomelano) [15], siendo el criptomelano el compuesto más estudiado y conocido. Además de estas familias túneles ( $m \times n)$, también se han descrito otro tipo de estructuras laminares y estructuras tipo túnel. La estructura de la espinela de Mn posee un túnel tri-dimensional (1x3). Se han sintetizado compuestos tipo espinelas de $\mathrm{Li}$ y $\mathrm{Mn}$ cuya fórmula general es $\mathrm{Li}_{\mathrm{n}} \mathrm{Mn}_{2-\mathrm{x}} \mathrm{O}_{4}(1 \leq \mathrm{n} \leq 1.33,0 \leq \mathrm{x} \leq 0.33$, $\mathrm{n} \geq 1+\mathrm{x})$, que presentan estructura tri-dimensional [8,16-22]. Asimismo, un gran número de trabajos indican las aplicaciones de las espinelas de Li-Mn como cátodos en baterías recargables [3, 17, 18, 23-26]. Estos óxidos de manganeso con estructura tipo túnel y tipo espinela pueden ser sintetizados a partir de sistemas laminares tipo birnesita $[3,17,18$, 25-27]. La formación de estos óxidos de manganeso con estructura túnel a partir de materiales laminares se considera que ocurre mediante el colapso de las láminas alrededor de los cationes localizados en el interior de los túneles formados, siendo las birnesitas materiales adecuados como precursores para la síntesis de óxidos de manganeso que presentan túneles en su estructura. Dependiendo del tamaño, estos cationes pueden actuar incluso como "plantillas" para materiales con distinto tipo de túnel [3].

En el presente trabajo se recogen resultados de la caracterización de los sólidos obtenidos al calcinar birnesitas con distintos cationes interlaminares preparados por el método sol-gel y sometidas a cambio iónico.

\section{EXPERIMENTAL}

\subsection{Preparación de las muestras}

\subsubsection{PREPARACIÓN DE LAS BIRNESITAS POTÁSICA Y SÓDICAS}

Se siguió el método propuesto por Ching y col. [9,10] para preparar birnesitas con potasio o sodio interlaminar. Sobre una disolución acuosa formada tras disolver $5.04 \mathrm{~g}$ de glucosa $(+)(0.40 \mathrm{M})$ en $70 \mathrm{~mL}$ de agua destilada se añadieron $100 \mathrm{~mL}$ de $\mathrm{KMnO}_{4}(0.27 \mathrm{M})$ o $100 \mathrm{~mL}$ de $\mathrm{NaMnO}_{4} \cdot \mathrm{H}_{2} \mathrm{O}(0.27 \mathrm{M})$, para preparar las birnesitas de $\mathrm{K}$ y Na, respectivamente, con relación molar glucosa/catión de 1; para la preparación de birnesita con relación molar glucosa/catión de 1.5 se utilizaron $20 \mathrm{~mL}$ de la disolución de glucosa $(+)(1.4 \mathrm{M})$ a la que se añadieron $50 \mathrm{~mL}$ de $\mathrm{NaMnO}_{4} \cdot \mathrm{H}_{2} \mathrm{O}(0.38 \mathrm{M})$. La mezcla resultante se agitó vigorosamente hasta la aparición del gel (unos $20 \mathrm{~s}$ para la de $\mathrm{K}$ y de 40 a 60 s para las de $\mathrm{Na}$ ) y seguidamente se dejó en reposo. La reacción es exotérmica, formándose inmediatamente un gel de color marrón, que libera agua, al tiempo que se contrae ligeramente; el agua se eliminó del medio mediante decantación durante un período de una hora, aproximadamente. A continuación el gel se secó en estufa a 110 ${ }^{\circ} \mathrm{C}$ durante una noche, obteniendo un xerogel de color marrón-negro. El xerogel se calcinó en la mufla al aire a $400{ }^{\circ} \mathrm{C}$ durante 2 horas. Se obtuvo de este modo un sólido negro-grisáceo con textura de algodón; el cual se lavó y secó a $110^{\circ} \mathrm{C}$ durante una noche.

\subsubsection{CAMBIO IÓNICO}

Este procedimiento se utilizó para la incorporación de $\mathrm{Cu}^{2+}, \mathrm{Mg}^{2+} \mathrm{o}$ $\mathrm{Li}^{+}$a la región interlaminar de birnesitas preparadas por el método solgel [28]. Para ello, se dispersaron $0.5 \mathrm{~g}$ de la birnesita en su forma sódica o potásica en $100 \mathrm{~mL}$ de disolución $1 \mathrm{M}$ de $\mathrm{M}\left(\mathrm{NO}_{3}\right)_{2} \cdot 4 \mathrm{H}_{2} \mathrm{O}(\mathrm{M}=\mathrm{Cu}$ y Mg) o LiCl. La suspensión se agitó magnéticamente durante 24 h, se lavó por centrifugación con varias porciones de agua destilada y los sólidos separados por filtración se secaron en estufa a $110{ }^{\circ} \mathrm{C}$ durante toda una noche.

\subsubsection{SISTEMAS CALCINADOS}

Todas las muestras preparadas se calcinaron a 600 y $1000{ }^{\circ} \mathrm{C} \mathrm{du}-$ rante 2 horas en mufla al aire.

\subsubsection{DESIGNACIÓN DE LAS MUESTRAS}

Para la designación de las muestras se ha seguido el siguiente código: muestra original $\mathrm{MnXY}(\mathrm{X}=$ catión interlaminar; $\mathrm{Y}=$ relación molar glucosa/catión en la síntesis); muestra intercambiada MnXYIZ ( $\mathrm{Z}=$ catión entrante); muestra calcinada MnXYIZ-T ( $\mathrm{T}=$ temperatura de calcinación en $\left.{ }^{\circ} \mathrm{C}\right)$.

\subsection{Caracterización de las muestras}

\subsubsection{ANÁLISIS QUÍMICO ELEMENTAL}

El contenido en metales fue determinado en el Servicio General de Análisis Químico Aplicado de la Universidad de Salamanca mediante Absorción Atómica, en un aparato Mark 2 ELL-240, tras disgregación de las muestras en ácido clorhídrico.

\subsubsection{DIFRACCIÓN DE RAYOS X}

Los difractogramas de rayos $\mathrm{X}$ en polvo (DRX) se han obtenido en un difractómetro Siemens modelo D500, con anticátodo de cobre y monocromador de grafito. Se utilizó la radiación $\mathrm{Cu} \mathrm{K \alpha}(\lambda=1.54050$ $\AA$ )), fijando la corriente en el filamento en $30 \mathrm{~mA}$, una ddp de $40 \mathrm{kV}$ y una velocidad de barrido (20) de $2 \%$ min. Para la identificación de los componentes cristalinos de las muestras, a partir de sus máximos de difracción, se utilizaron las fichas existentes en la base de datos JCPDS (Joint Committee on Powder Diffraction Standards) [29].

\subsubsection{ANÁLISIS TÉRMICOS}

Los estudios térmicos han sido realizados en los equipos PerkinElmer DTA7 y Perkin-Elmer TG7, ambos conectados a un ordenador Dell y utilizando el software Pyris, suministrado también por PerkinElmer. Los análisis se llevaron a cabo en atmósfera dinámica de oxígeno $(20 \mathrm{~mL} / \mathrm{min})$ y una velocidad de calentamiento de $10{ }^{\circ} \mathrm{C} / \mathrm{min}$.

\subsubsection{ESPECTROSCOPÍA INFRARROJA CON TRANSFOR- MADA DE FOURIER (FT-IR)}

Los espectros FT-IR de las muestras se obtuvieron por la técnica de pastilla de $\mathrm{KBr}$ y se registraron en un espectrómetro Perkin-Elmer FT1730, en el intervalo $4000-400 \mathrm{~cm}^{-1}$, registrando 100 barridos (para mejorar la relación señal/ruido) con una resolución nominal de $2 \mathrm{~cm}^{-1}$.

\subsubsection{SUPERFICIE ESPECÍFICA Y POROSIDAD}

La superficie específica de las muestras, así como sus características de textura superficial, se determinaron a partir de las isotermas de adsorción-desorción de nitrógeno a $-196{ }^{\circ} \mathrm{C}$, que se registraron en un aparato Gemini, de Micromeritics. Las muestras $(0.1 \mathrm{~g})$ fueron previamente desgasificadas por calentamiento a $150{ }^{\circ} \mathrm{C}$ durante $2 \mathrm{~h}$ en flujo 
de $\mathrm{N}_{2}$ en un aparato FlowPrep 060, de Micromeritics. La superficie específica fue determinada por el método BET [30]. El análisis de las isotermas se llevó a cabo con el programa SAPO 4.5 desarrollado en nuestro laboratorio [31].

\subsubsection{MICROSCOPÍA ELECTRÓNICA DE BARRIDO}

Las microfotografías SEM se obtuvieron en un microscopio JEOL 6300, con una tensión de $25 \mathrm{kV}$ y una distancia a muestra de $8 \mathrm{~mm}$. Las muestras se prepararon por dispersión en acetona, deposición de unas gotas sobre una portamuestras de cobre y recubrimiento con una capa de oro con un sombreador de oro BALTEC SCD005 mediante sputtering.

\section{RESULTADOS Y DISCUSION}

\subsection{Precursores: birnesitas}

En la Tabla 1 se incluyen las fórmulas correspondientes a las muestras originales, calculadas a partir de los resultados de análisis químico elemental, refiriendo la fórmula estructural a una estequiometría próxima a $\mathrm{MnO}_{2}$. Se observa que la cantidad de sodio (en expresión molar) incorporada es casi un $50 \%$ superior a la de potasio, hecho que puede estar relacionado con la turbidez previa a la formación del sol o con el menor radio iónico del $\mathrm{Na}^{+}$, que puede así acceder con mayor facilidad a la región interlaminar, aunque las diferencias de espaciado no son significativas en los difractogramas de rayos $\mathrm{X}$ de los difractogramas originales. El contenido en agua se ha calculado a partir de la pérdida de peso registrada en las dos primeras etapas de los diagramas termogravimétricos [32], que llega hasta aproximadamente unos 400-500 ${ }^{\circ} \mathrm{C}$, ya que hasta dicha temperatura las birnesitas, preparadas por el método sol-gel, son estables.

Tabla 1.-Resultados de análisis químico elemental y valores de superficie específica de las muestras originales e intercambiadas.

\begin{tabular}{|c|c|c|c|c|c|}
\hline Muestra & $\mathrm{Mn}^{*}$ & $\mathrm{~K}^{*} / \mathrm{Na}^{*}$ & $\mathrm{~A}^{*}$ & Fórmula & $\mathrm{S}_{\mathrm{BET}}\left(\mathrm{m}^{2} / \mathrm{g}\right)$ \\
\hline MnK1 & 55.4 & 7.5 & & $\mathrm{~K}_{0.190} \mathrm{MnO}_{2} \cdot 0.35 \mathrm{H}_{2} \mathrm{O}$ & 16 \\
\hline MnK1IMg & 54.9 & 5.2 & 0.72 & $\mathrm{~K}_{0.132} \mathrm{Mg}_{\mathrm{g}_{0.030}} \mathrm{MnO}_{2} \cdot 0.51 \mathrm{H}_{2} \mathrm{O}$ & 19 \\
\hline $\mathrm{MnK} 1 \mathrm{ICu}$ & 53.9 & 1.4 & 4.84 & $\mathrm{~K}_{00036} \mathrm{Cu}_{0078} \mathrm{MnO}_{2} \cdot 0.71 \mathrm{H}_{2} \mathrm{O}$ & 27 \\
\hline MnK1ILi & 57.2 & 0.47 & 1.3 & $\mathrm{~K}_{0.012} \mathrm{Li}_{0.180} \mathrm{MnO}_{2} \cdot 0.77 \mathrm{H}_{2} \mathrm{O}$ & 44 \\
\hline MnNa1 & 50.9 & 7.04 & & $\mathrm{Na}_{0.30} \mathrm{MnO}_{2} \cdot 0.91 \mathrm{H}_{2} \mathrm{O}$ & 29 \\
\hline MnNa1IMg & 52.2 & 3.5 & 2.0 & $\mathrm{Na}_{0.160} \mathrm{Mg}_{0.08} \mathrm{MnO}_{2} \cdot 0.97 \mathrm{H}_{2} \mathrm{O}$ & 33 \\
\hline $\mathrm{MnNa} 1 \mathrm{ICu}$ & 49.5 & 0.24 & 9.2 & $\mathrm{Na}_{0.012} \mathrm{Cu}_{0.160} \mathrm{MnO}_{2} \cdot 1.27 \mathrm{H}_{2} \mathrm{O}$ & 33 \\
\hline MnNa1ILi & 53.1 & 0.1 & 2.2 & $\mathrm{Na}_{0.005} \mathrm{Li}_{0.325} \mathrm{MnO}_{2} \cdot 0.90 \mathrm{H}_{2} \mathrm{O}$ & 60 \\
\hline MnNa1.5 & 51.8 & 7.2 & & $\mathrm{Na}_{0.330} \mathrm{MnO}_{2} \cdot 0.70 \mathrm{H}_{2} \mathrm{O}$ & 32 \\
\hline MnNa1.5IMg & 49.8 & 2.9 & 2.1 & $\mathrm{Na}_{0.138} \mathrm{Mg}_{0.096} \mathrm{MnO}_{2} \cdot 0.99 \mathrm{H}_{2} \mathrm{O}$ & 37 \\
\hline $\mathrm{MnNa} 1.5 \mathrm{ICu}$ & 47.4 & 0.14 & 8.8 & $\mathrm{Na}_{0.007} \mathrm{Cu}_{0.161} \mathrm{MnO}_{2} \cdot 1.03 \mathrm{H}_{2} \mathrm{O}$ & 33 \\
\hline MnNa1.5ILi & 52.3 & 0.20 & 2.1 & $\mathrm{Na}_{0.009} \mathrm{Li}_{0.321} \mathrm{MnO}_{2} \cdot 0.87 \mathrm{H}_{2} \mathrm{O}$ & 55 \\
\hline
\end{tabular}

*\% peso; $\mathrm{A}=\mathrm{Mg}, \mathrm{Cu}, \mathrm{Li}$

Estas tres muestras se sometieron a intercambio iónico con los cationes $\mathrm{Cu}^{2+}, \mathrm{Mg}^{2+} \mathrm{o} \mathrm{Li}^{+}$[33]. En ningún caso se alcanza un intercambio completo de los iones sodio o potasio iniciales, aunque el sodio se intercambia en mayor medida que el potasio.

Los difractogramas de estas muestras sintéticas tipo birnesita presentan dos máximos característicos a $\approx 7 \AA$ (001) y a $3.5 \AA$ (002) que son más agudos e intensos que los de las muestras naturales, indicando una menor cristalinidad en estas últimas [10]. En las muestras aquí preparadas, el primer máximo se registra a 7.14, 7.18 y $7.15 \AA ̊$ para las muestras MnK1, MnNa1 y MnNa1.5, respectivamente, y el segundo a
3.5 Å en todos los casos (Figura 2). Además, se registran otros máximos con intensidades mucho más débiles. En todos los casos, el máximo próximo a $7 \AA$ es el más característico de estos materiales, y corresponde a la distancia lámina-lámina entre dos láminas consecutivas de octaedros $\left[\mathrm{MnO}_{6}\right]$ que comparten aristas. El valor de espaciado de este máximo variará, en mayor o menor medida, con el tamaño del catión interlaminar y el grado de hidratación, así como con el grado de no-estequiometría que el compuesto presente. En las muestras intercambiadas se mantiene la estructura laminar de la muestra de partida, con máximos característicos de la estructura laminar tipo birnesita centrados a $\approx 7 \mathrm{y} \approx 3.5 \AA$ A para los planos de difracción (001) y (002), respectivamente. Únicamente se observan ligeras variaciones en la posición del máximo de difracción (001) para los distintos cationes intercambiados.

En los espectro FT-IR se registra, a bajos números de ondas, tanto de las muestras originales como las intercambiadas, una banda intensa y ancha centrada, con muy ligeras variaciones, a $525 \mathrm{~cm}^{-1}$, con un hombro agudo próximo a $470 \mathrm{~cm}^{-1}$, junto a otro hombro, más débil, próximo a $425 \mathrm{~cm}^{-1}$, que es característico de la birnesita y discrimina ésta de otros óxidos laminares de manganeso. Todos los óxidos de manganeso laminares conocidos presentan una intensa banda entre $450-400 \mathrm{~cm}^{-1}$ [34].

Son muestras no microporosas cuya superficie específica aumenta al someterlas a cambio iónico, lo que indica que el cambio actúa como un proceso de envejecimiento, alterando la superficie de estos sólidos.

\subsection{Sistemas Calcinados}

Tras calcinar a $600{ }^{\circ} \mathrm{C}$ se observa en todos los casos la pérdida de la estructura laminar, formándose compuestos con estructuras bien definidas. En el difractograma de la muestra MnK1-600 (Figura 3) se registran únicamente picos correspondientes a la fase criptomelano [estructura túnel (2x2)]. Ching y col. [10] indican que la transformación desde la estructura laminar a la estructura túnel ocurre mediante el colapso de la estructura alrededor del catión interlaminar y que el tamaño del $\mathrm{K}^{+}$es adecuado para la formación de la estructura de criptomelano. Golden y col. [25] encuentran que el estado de desorden se produce, en algunos casos, a temperaturas relativamente bajas (200$400{ }^{\circ} \mathrm{C}$ ), pero el criptomelano no se detecta hasta que la temperatura

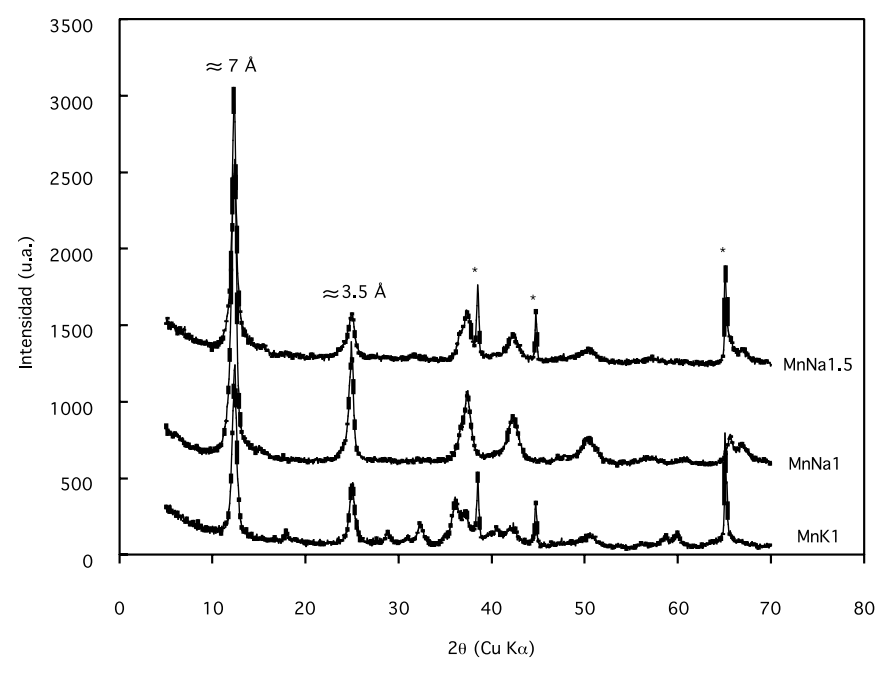

Figura 2.- Difractogramas de rayos $\mathrm{X}$ de las muestras originales. Los máximos marcados $\left({ }^{*}\right)$ corresponden al portamuestras de aluminio. 
de calcinación es de $600{ }^{\circ} \mathrm{C}$. Dado que las muestras preparadas por este método se han calcinado previamente a $400{ }^{\circ} \mathrm{C}$ para que adopten la estructura tipo birnesita, su estructura tiene que ser estable, por lo menos, hasta esta temperatura. No obstante, en muestras preparadas por métodos distintos al sol-gel el criptomelano se ha detectado al calcinar a $6000^{\circ} \mathrm{C}[25,27]$.

Como se ha indicado anteriormente, el proceso de cambio iónico no es completo en las condiciones de trabajo utilizadas, por lo que la presencia de pequeñas cantidades de potasio en todas las muestras da lugar a la formación de la fase criptomelano, junto con otras fases características del catión incorporado. En la Figura 3 se incluyen los difractogramas de rayos $X$ de las muestras calcinadas obtenidas a partir de la original de potasio. A efectos comparativos se han incluido también los difractogramas de las muestras originales.

En el difractograma de la muestra MnK1ILi-600 se identifica la espinela de $\mathrm{Li}, \mathrm{LiMn}_{2} \mathrm{O}_{4^{\prime}}$ junto con pequeñas cantidades de criptomelano y bixbita. Para la muestra MnK1IMg se observa un comportamiento térmico muy parecido al de la birnesita original, MnK1, formándose a $600{ }^{\circ} \mathrm{C}$ el criptomelano y una pequeña cantidad de óxido mixto $\mathrm{Mn}$ Mg (JCPDS: 23-392) [29], dado que el $\mathrm{Mg}^{2+}$ sustituye en muy pequeña proporción al $\mathrm{K}^{+}$. El compuesto $\mathrm{MgO} \cdot \mathrm{Mn}_{2} \mathrm{O}_{3}$ es un óxido mixto $\mathrm{Mn}-\mathrm{Mg}$ con estructura tetragonal. Por último, en el difractograma de rayos $\mathrm{X}$ de la muestra $\mathrm{MnK} 1 \mathrm{ICu}-600$ se detecta la presencia de estructuras tipo espinela de $\mathrm{Cu}\left(\mathrm{Cu}_{1.5} \mathrm{Mn}_{1.5} \mathrm{O}_{4}\right)$, en pequeña proporción, junto a mezclas de criptomelano y bixbita.

La calcinación a $1000{ }^{\circ} \mathrm{C}$ de la muestra MnK1 conduce a la transformación de parte de la estructura túnel $(2 \times 2)$, formada a $600{ }^{\circ} \mathrm{C}$, en hausmanita, $\mathrm{Mn}_{3} \mathrm{O}_{4}$. En una muestra potásica sintetizada por el método sol-gel, Ching y col. [10] obtienen criptomelano al calcinar a $600{ }^{\circ} \mathrm{C}$, y a $800{ }^{\circ} \mathrm{C}$ se produce una pequeña degradación hasta $\mathrm{Mn}_{3} \mathrm{O}_{4}$ (hausmanita), pero aun observan fases de estructura laminar a esta temperatura y ya a $1000^{\circ} \mathrm{C}$ se obtiene la degradación total a hausmanita. En los difractogramas de rayos X de las muestras MnK1IMg-1000 y MnK1ICu-1000 se observan los máximos de difracción correspondientes a la fase hausmanita, mientras que en el difractograma de la muestra MnK1ILi-1000 se detecta la presencia mayoritaria de la espinela de Li, con picos débiles debidos a hausmanita.

En todos los difractogramas de las muestras sódicas calcinadas se observa la formación de nuevos compuestos, perdiéndose también en este caso la estructura laminar tras calcinar a $600^{\circ} \mathrm{C}$. En algunos de los difractogramas de esta serie los máximos son anchos, lo que indica que las fases formadas no son tan cristalinas como en el caso de las obtenidas a partir de las muestras potásicas; los difractogramas no son tan "limpios", registrándose muchos picos de baja intensidad, debido a la gran variedad de compuestos de estequiometría próxima que forma el sodio con el manganeso y oxígeno [35]. La relación glucosa/catión no modifica las fases cristalinas presentes en los sólidos obtenidos tras la calcinación, observándose únicamente ligeras variaciones en la intensidad o la anchura de los picos de las distintas fases formadas.

En la Figura 4 se recogen los difractogramas de rayos $\mathrm{X}$ de las muestras originales y calcinadas obtenidas a partir de la que contiene sodio inicialmente. En el difractograma de las muestra MnNa1-600 se han identificado mayoritariamente fases cuyas fórmulas estructurales corresponden a $\mathrm{Na}_{2} \mathrm{Mn}_{5} \mathrm{O}_{10} \cdot \mathrm{nH}_{2} \mathrm{O}$ y $\mathrm{Na}_{0.2} \mathrm{MnO}_{2}$. La fase $\mathrm{Na}_{2} \mathrm{Mn}_{5} \mathrm{O}_{10}$ $\left(\mathrm{Na}_{0.4} \mathrm{MnO}_{2}\right)$ es una estructura tipo túnel $(2 \times 3)$ de la familia de la romanechita [8]. El compuesto $\mathrm{Na}_{0.2} \mathrm{MnO}_{2}$ ha sido identificado por Parant y col. [35] como perteneciente a la familia de las holanditas, con estructura túnel (2×2), y cuyo tamaño de túnel es mucho menor del habitual debido al menor tamaño del catión $\mathrm{Na}^{+}$, con respecto al de $\mathrm{K}^{+}$. Para las muestras sódicas intercambiadas se obtienen resultados prácticamente iguales para ambas series, independientemente de la relación glucosa / catión utilizada, como era de esperar a la vista de los resultados obte-

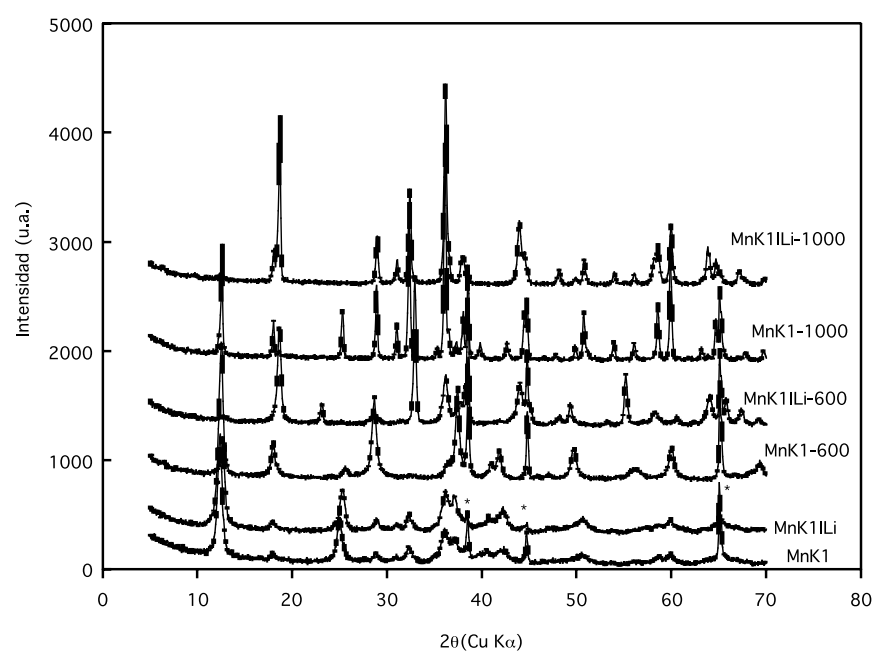

Figura 3.-Difractogramas de rayos $\mathrm{X}$ de las muestras intercambiadas de K sometidas a calcinación. Los máximos marcados $\left(^{*}\right)$ corresponden al portamuestras de aluminio.

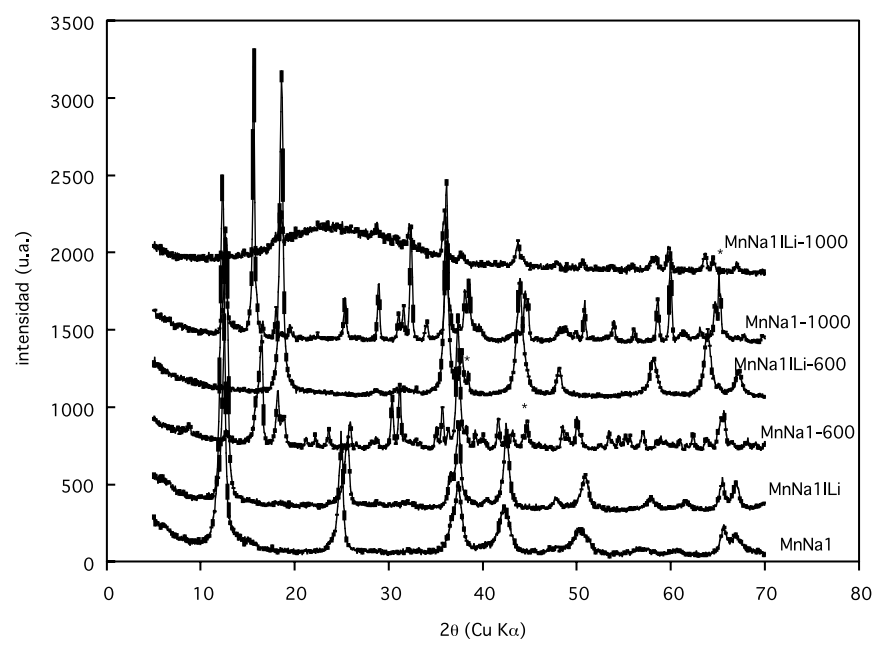

Figura 4.-Difractogramas de rayos $\mathrm{X}$ de las muestras intercambiadas de Na sometidas a calcinación. Los máximos marcados $\left(^{*}\right)$ corresponden al portamuestras de aluminio.

nidos para las muestras originales no calcinadas. El alto porcentaje de intercambio, con respecto a la de potasio, hace que, en algunos casos, presenten un comportamiento distinto al que tienen las de potasio, no observándose, tras calcinar, óxidos mixtos de manganeso y sodio. Así, los difractogramas de las muestras MnNa1ILi-600 y MnNa1.5ILi-600 presentan máximos agudos e intensos característicos de una fase bien cristalizada, que se ha identificado como la espinela $\mathrm{LiMn}_{2} \mathrm{O}_{4}$. A pesar de que para la muestra MnK1ILi el porcentaje de intercambio es del 94 $\%$, la alta tendencia del $\mathrm{K}^{+}$a formar criptomelano daba lugar a que en la muestra MnK1ILi-600 se observara, junto con la espinela $\mathrm{LiMn}_{2} \mathrm{O}_{4^{\prime}}$ los compuestos criptomelano y bixbita. En el difractograma de la muestra MnNa1IMg-600 se registran máximos debidos a la formación de compuestos $\mathrm{Mn}-\mathrm{Na}$, identificados como $\mathrm{Na}_{2} \mathrm{Mn}_{8} \mathrm{O}_{16}\left(\mathrm{Na}_{0.125} \mathrm{MnO}_{2}\right)$ y $\mathrm{Na}_{2} \mathrm{Mn}_{5} \mathrm{O}_{10}\left(\mathrm{Na}_{04} \mathrm{MnO}_{2}\right)$ y, en pequeña proporción, un óxido mixto $\mathrm{Mn}-\mathrm{Mg}$ con celdilla unidad tetragonal (JCPDS 23-392 [29]). La fase $\mathrm{Na}_{2} \mathrm{Mn}_{8} \mathrm{O}_{16}$ es una estructura túnel tipo holandita [8]. Para las muestras MnNa1ICu-600 y MnNa1.5ICu-600 se observan casi exclusivamente los picos correspondientes al compuesto $\mathrm{Mn}_{2} \mathrm{O}_{3}$ (bixbita), junto con pequeñas cantidades de $\mathrm{Na}_{2} \mathrm{Mn}_{8} \mathrm{O}_{16}$ y la espinela de $\mathrm{Cu}\left(\mathrm{Cu}_{1.5} \mathrm{Mn}_{1.5} \mathrm{O}_{4}\right)$. 
$\mathrm{Al}$ calcinar a $1000{ }^{\circ} \mathrm{C}$ las muestras sódicas (MnNa1 y MnNa1.5) evolucionan principalmente a $\mathrm{Na}_{0.2} \mathrm{MnO}_{2}$ y $\mathrm{Na}_{0.7} \mathrm{MnO}_{2.05^{\prime}}$ observándose otros picos más agudos e intensos correspondientes a la espinela normal de manganeso distorsionada tetragonalmente $\left(\mathrm{Mn}_{3} \mathrm{O}_{4}\right)$. Para las muestras sódicas intercambiadas con $\mathrm{Cu}^{2+} \mathrm{y} \mathrm{Mg}^{2+}(\mathrm{MnNa} 1 \mathrm{ICu}$, MnNa1.5ICu, MnNa1IMg y MnNa1.5IMg) se observa al calcinar a $1000^{\circ} \mathrm{C}$ la aparición de picos de difracción intensos y agudos pertenecientes a hausmanita; en cambio, para las muestras con $\mathrm{Li}^{+}(\mathrm{MnNa} 1 \mathrm{ILi}$ y MnNa1.5ILi) se obtiene la mezcla de espinela de manganeso (hausmanita) y la espinela de $\mathrm{Li}\left(\mathrm{LiMn}_{2} \mathrm{O}_{4}\right)$, cuyos picos son más anchos, indicando una débil cristalización.

En la Figura 5 se han incluído los espectros FT-IR de algunas muestras seleccionadas. En el espectro FT-IR de la muestra MnK1-600 se observa una banda ancha y débil, a aproximadamente $\approx 3500 \mathrm{~cm}^{-1}$, y otra banda también débil cerca de $1600 \mathrm{~cm}^{-1}$, ambas debidas a la existencia de moléculas de agua; la anchura y debilidad de las bandas indica que la cantidad de agua es poca y que no ocupan sitios cristalográficos definidos [34]. A más bajos números de onda se observa, Figura 5, claramente bandas a $690,675,583$ y $529 \mathrm{~cm}^{-1}$, características de las vibraciones $\mathrm{Mn}-\mathrm{O}$ en la especie criptomelano [34]. En el espectro de la muestra calcinada a $1000^{\circ} \mathrm{C}$ se observan nuevas bandas centradas a $\approx 621,524,422$ y $358 \mathrm{~cm}^{-1}$ características de los modos de vibración $v_{1^{\prime}}$ $v_{2^{\prime}} v_{3} y_{4^{\prime}}$, representativos de los enlaces Mn-O de la espinela de manganeso $\left(\mathrm{Mn}_{3} \mathrm{O}_{4}\right)$, junto a otras bandas más débiles, indicando que aún existe algo de la fase criptomelano a esta temperatura. Los espectros FT-IR de las muestras MnK1IMg-600 y MnK1ICu-600 son similares al de la muestra MnK1-600, ya que en ellos coexiste la fase criptomelano junto a óxidos de los cationes intercambiados.

Los espectros de las muestras MnNa1, MnNa1IMg y MnNa1ICu calcinadas a $600{ }^{\circ} \mathrm{C}$ (no incluídos en la figura) son bastante similares, ya que en todas ellas existen compuestos de manganeso y sodio con estructura túnel. Se observan bandas debidas a las vibraciones del enlace Mn-O de los óxidos mixtos de manganeso con estructura túnel, alguno de ellos pertenecientes a la familia de las holanditas, identificados por difracción de rayos X; dichas bandas están mejor resueltas a medida que aumenta la cristalinidad de los compuestos. En todos los casos se observa una pequeña banda $\mathrm{a} \approx 330 \mathrm{~cm}^{-1}$, así como una banda prominente $\mathrm{a} \approx 570 \mathrm{~cm}^{-1}$, que son específicas de las vibraciones $\mathrm{Mn}-\mathrm{O}$ de los minerales de la familia de las holanditas [34]; esta última banda $\left(\approx 600 \mathrm{~cm}^{-1}\right)$ aparece como un pequeño hombro en la romanechita [34]. En el espectro de la muestra MnNa1 calcinada a $600{ }^{\circ} \mathrm{C}$ sí se observa un pequeño hombro $\mathrm{a} \approx 617 \mathrm{~cm}^{-1}$, debido a que a dicha temperatura se forma algo de romanechita, según indicaban los datos de difracción de rayos X. En el espectro FT-IR de la muestra MnNa1ILi-600 (Figura 5) se observan solamente dos bandas bien definidas y pronunciadas $\mathrm{a} \approx$ 617 y $516 \mathrm{~cm}^{-1}$ debidas a los modos vibracionales $v_{2}$ y $v_{4}(\mathrm{Mn}-\mathrm{O})$ de la espinela de $\mathrm{Li}\left(\mathrm{LiMn}_{2} \mathrm{O}_{4}\right)$ [19]. Asimismo, se observa la banda débil a $\approx$ $330 \mathrm{~cm}^{-1}$ característica de las vibraciones $\mathrm{Mn}-\mathrm{O}$ de los minerales de la familia de las holanditas [34]. En los espectros FT-IR de las muestras calcinadas a $1000{ }^{\circ} \mathrm{C}$ sólo se observan las cuatro bandas debidas a las vibraciones $\mathrm{Mn}-\mathrm{O}$ de la hausmanita $\left(\mathrm{Mn}_{3} \mathrm{O}_{4}\right)$.

Por último, en la Figura 6 se representa la variación del valor de $\mathrm{S}_{\mathrm{BET}}\left(\mathrm{m}^{2} / \mathrm{g}\right)$ frente a la temperatura de calcinación. De forma general, se observa una fuerte disminución del valor de $\mathrm{S}_{\mathrm{BET}}\left(\mathrm{m}^{2} / \mathrm{g}\right)$ al aumentar la temperatura de calcinación, debido a la formación de nuevas fases muy cristalinas; a $1000{ }^{\circ} \mathrm{C}$ ninguna de estas muestra presenta valores de superficie apreciables ya que los valores medidos están por debajo de $1 \mathrm{~m}^{2} / \mathrm{g}$. Para la serie MnK1 todas las muestras calcinadas a $600{ }^{\circ} \mathrm{C}$ tienen valores de $S_{B E T}$ muy similares, posiblemente debido a que en todas ellas se forma criptomelano a dicha temperatura, por la alta tendencia que tiene el $\mathrm{K}^{+}$a formar dicha fase cuando se destruye la fase laminar. Para las series MnNa1 y MnNa1.5 se observan diferencias
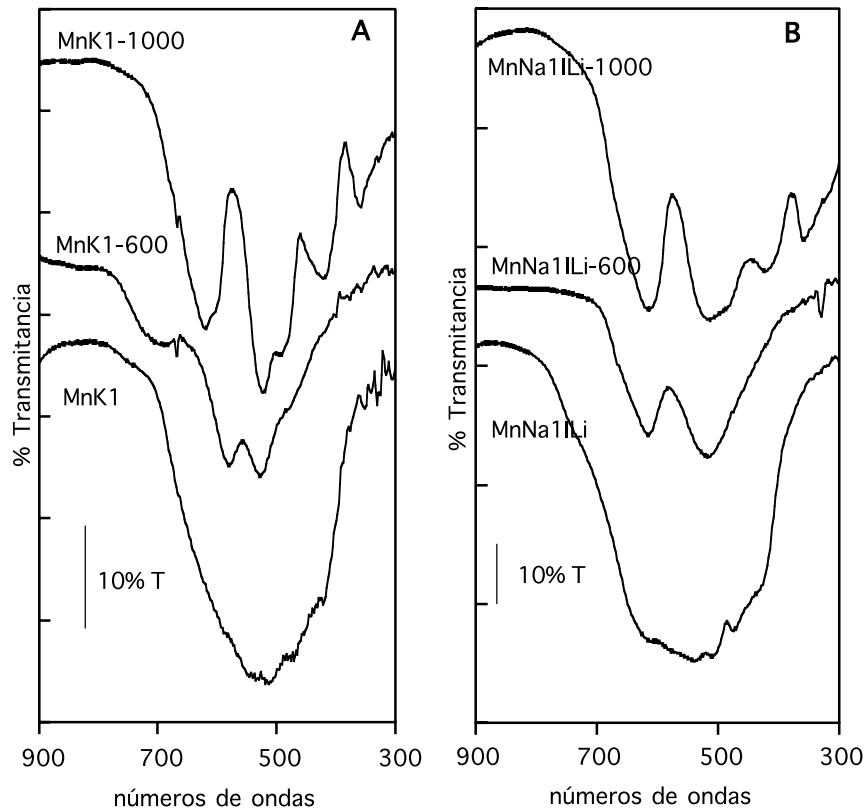

Figura 5.-Espectros FT-IR de las muestras MnK1 y MnNa1-Li originales y calcinadas.
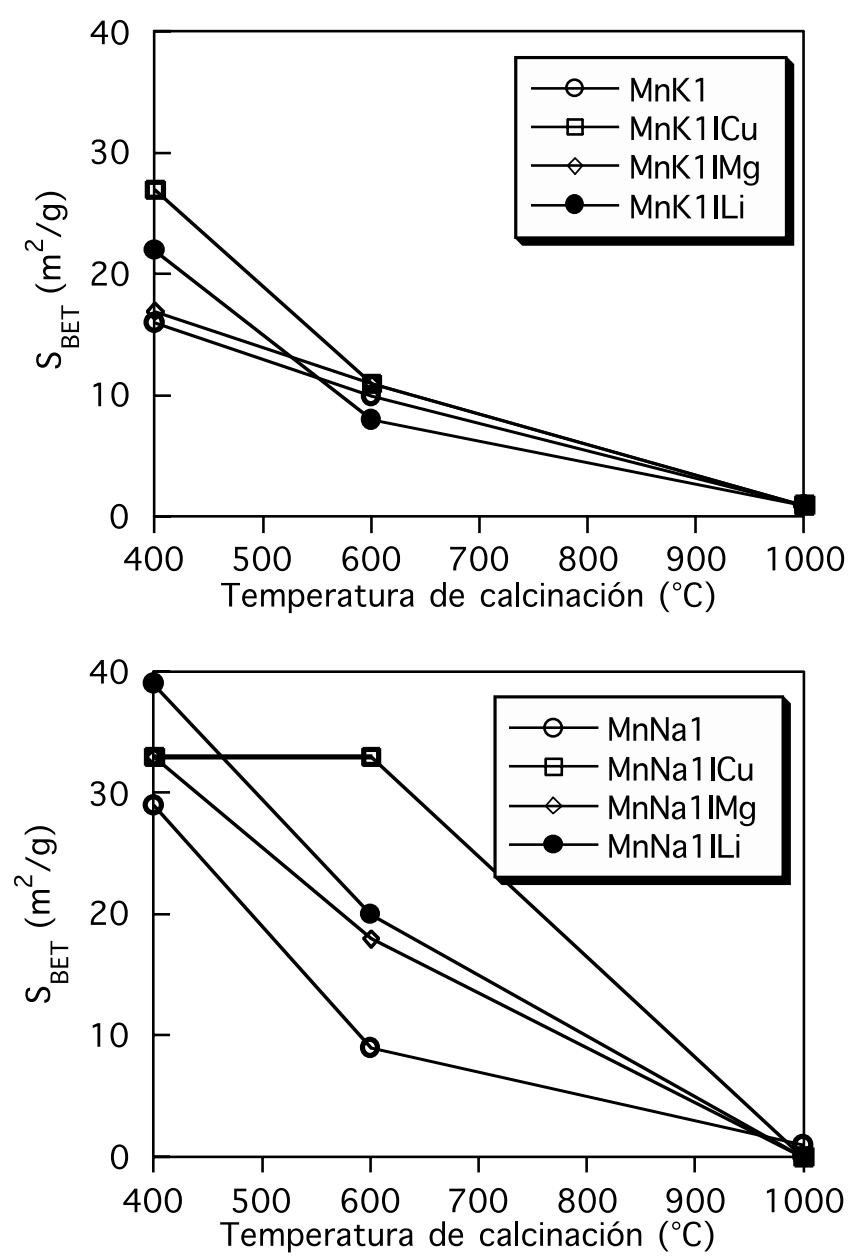

Figura 6.-Variaciones en la superficie BET de las muestras con la temperatura de calcinación. 
notables en los valores de $\mathrm{S}_{\mathrm{BET}}$ de las muestras calcinadas a $600^{\circ} \mathrm{C}$. Las muestras originales MnNa1 y MnNa1.5 tienen valores de superficie específica similares y sus correspondientes calcinadas también. No ocurre así con las muestras intercambiadas y calcinadas; mientras que en la serie $\mathrm{MnNa} 1$, es la muestra con $\mathrm{Cu}^{2+}$ la que presenta mayor valor de $\mathrm{S}_{\mathrm{BET}}$ a $600{ }^{\circ} \mathrm{C}$, en la serie $\mathrm{MnNa} 1.5$ es la muestra con $\mathrm{Li}^{+}$. Estas diferencias entre las dos series están relacionadas probablemente con la cristalinidad de las fases obtenidas. La presencia de fases amorfas da lugar a mayor superficie específica. La calcinación a $1000{ }^{\circ} \mathrm{C}$ produce, en todos los casos, una drástica disminución de la superficie específica debido a la formación de fases cristalinas, casi exclusivamente hausmanita $\left(\mathrm{Mn}_{3} \mathrm{O}_{4}\right)$.

Las microfotografías SEM de las muestras MnK1, MnK1-600 y MnNa1.5ILi-600 ponen de manifiesto la transformación superficial que se ha producido en dichas muestras con la calcinación. La muestra MnK1 presenta una morfología irregular en forma de láminas, junto con una forma redondeada debida probablemente al $\mathrm{Mn}_{2} \mathrm{O}_{3}$ identificado por rayos X. La muestra MnK1-600, identificada por XRD como criptomelano, presenta una morfología diferente a la de la muestra original, sin el desarrollo de poros grandes en la superficie, con aspecto de aglomerado y alguna forma redondeada. La muestra MnNa1.5ILi600 en la que, según indica el difractograma de rayos $X$, ha tenido lugar la formación mayoritaria de la espinela de $\mathrm{Li}\left(\mathrm{LiMn}_{2} \mathrm{O}_{4}\right)$, presenta una morfología similar a la descrita por otros autores para dicho compuesto [21].

\section{CONCLUSIONES}

Se han obtenido birnesitas cristalinas con Na y K en la interlámina por el método sol-gel; aunque el intercambio iónico no ha sido completo se ha alcanzado un grado de intercambio iónico prácticamente completo en el caso de las muestras con $\mathrm{Na}^{+}$por $\mathrm{Li}^{+}$. El intercambio iónico se produce topotácticamente manteniendo la estructura laminar de partida afectando, dicho tratamiento, a la superficie de los sólidos y actuando como un proceso de envejecimiento.

Son muestras estables térmicamente hasta una temperatura de 500-600 ${ }^{\circ} \mathrm{C}$; a partir de esta temperatura se produce la transformación de fase laminar a estructuras túnel o espinelas dependiendo del catión situado en la interlámina junto a moléculas de agua.

\section{BIBLIOGRAFÍA}

1. Q. Feng, H. Kanoh, Y. Mijay y K. Ooi. “Hydrotermal synthesis of lithium and sodium manganese oxides and their metal ion extraction/insertion reactions". Chem. Mater., 7, 1226-1232 (1995).

2. Q. Feng, H. Kanoh, Y. Mijay y K. Ooi. "Metal ion extraction/insertion reactions with todorokite-type manganese oxide in the aqueous phase". Chem. Mater., 7, 1722-1727 (1995)

3. Q. Feng, K. Yanagisawa y N. Yamasaki. "Transformation of manganese oxides from layered structures to tunnel structures". J. Chem. Soc., Chem. Comm., 1607-1608 (1996).

4. Q. Feng, K. Yanagisawa y N. Yamasaki. "Synthesis of birnessite-type lithium manganese oxide". J. Ceram. Soc. Jpn., 104, 897-899 (1996).

5. Q. Feng, E. H. Sun, K. Yanagisawa y N. Yamasaki. “Synthesis of birnessitetype sodium manganese oxides by solution reaction and hydrothermal methods". J. Ceram. Soc. Jpn., 105, 564-568 (1997).

6. Q. Feng, K. Yanagisawa y N. Yamasaki. "Synthesis of birnessite-type potassium manganese oxide". J. Mater. Sci. Lett., 16, 110-112 (1997).

7. Q. Feng, K. Yanagisawa y N. Yamasaki. “Hydrothermal soft chemical process for synthesis of manganese oxides with tunnel structures". J. Porous Mater., 5, 153-161 (1998).

8. Q. Feng, H. Kanoh y K. Ooi. “Manganese oxide porous crystals". J. Mater. Chem., 9, 319-333 (1999).
9. S. Ching, J. A. Landrigan, M. L. Jorgensen, N. Duan y S. L. Suib. "Sol-gel synthesis of birnessite from $\mathrm{KMnO}_{4}$ and simple sugars". Chem. Mater., 7, 1604-1606 (1995).

10. S. Ching, D. J. Petrovay, M. L. Jorgensen y S. L. Suib. "Sol-gel synthesis of layered birnessite-type manganese oxides". Inorg. Chem., 36, 883-890 (1997).

11. S. Ching, S. Roark, J. L. Duan y S. L. Suib. "Sol-gel route to the tunneled manganese oxide cryptomelane". Chem. Mater., 9, 750-754 (1997).

12. N. Yamamoto y O. Tamada. "Hydrothermal crystal growth of a new manganese dioxide". J. Crystal Growth, 73, 199-200 (1985).

13. T. Rziha, H. Gies y J. Rius. "RUB-7, a new synthetic manganese oxide structure type with a 2x4 tunnel". Eur. J. Mineral., 8 675-686 (1996).

14. P. Boullay, M. Hervieu y B. Raveau. "A new manganite with an original composite tunnel structure: $\mathrm{Ba}_{6} \mathrm{Mn}_{24} \mathrm{O}_{48}$ ". J. Solid State Chem., 132, 239-248 (1997).

15. S. R. Randall, D. M. Sherman y K. V. Ragnarsdottir. “An extended X-ray absorption fine structure spectroscopy investigation of cadmium sorption on cryptomelane $\left(\mathrm{KMn}_{8} \mathrm{O}_{16}\right)^{\prime \prime}$. Chem. Geol., 151, 95-105 (1998).

16. H. Berg, K. Göransson, B. Noläng y J. O. Thomas. “Electronic structure and stability of $\mathrm{Li}_{1} \mathrm{Mn}_{2} \mathrm{O}$ spinels". J. Mater. Chem., 10, 1437-1441 (2000).

17. Y. K. Sun y S. H. Jin. "Synthesis and electrochemical characteristics of spinel phase $\mathrm{LiMn}_{2} \mathrm{O}_{4}$-based cathode materials for lithium polymer batteries". J. Mater. Chem., 8, 2399-2404 (1998).

18. A. R. Armstrong, H. Huang, R. A. Jennigs y P. G. Bruce. " $\mathrm{Li}_{0.44} \mathrm{MnO}_{2}$ : an intercalation electrode with a tunnel structure and excellent cyclability". J. Mater. Chem., 8, 255-259 (1998).

19. S. R. S. Prabaharan, S. S. Michael y C. Julien. “Synthesis and electrochemistry of $\mathrm{LiMn}_{2} \mathrm{O}_{4}$ prepared using succinic acid as complexing agent". Int. J. Inorg. Mater., 1, 21-27 (1999).

20. E. N. Zhecheva, M. Y. Gorova y R. K. Stoyanova.. "Microstructure of $\mathrm{Li}_{1+x} \mathrm{Mn}_{2-\mathrm{x}} \mathrm{O}_{4}$ spinels obtained from metal-organic precursors". J. Mater. Chem., 9, 1559-1567 (1999).

21. X. Yang, H. Kanoh, W. Tang y K. Ooi. “Synthesis of $\mathrm{Li}_{1.33} \mathrm{Mn}_{1.67} \mathrm{O}_{4}$ spinels with different morphologies and their ion adsorptivities after delithiation". J. Mater. Chem., 10, 1903-1909 (2000).

22. J. Akimoto, Y. Takahashi, Y. Gotoh y S. Mizuta. "Single crystal X-ray diffraction study of the spinel-type $\mathrm{LiMn}_{2} \mathrm{O}_{4}{ }^{\prime \prime}$. Chem. Mater., 12, 3246-3248 (2000).

23. J. R. Dahn, U. V. Sacken, M. W. Juzkow y H. Al-Janaby. "Rechargeable $\mathrm{LiNiO}_{2}$ / carbon cells". J. Electrochem. Soc., 138, 2207-2211 (1991).

24. M. H. Rossouw, D. C. Liles, M. M. Thackeray, W. I. F. David y S. Hull. “Alpha manganese dioxide for lithium batteries: A structural and electrochemical study". Mater. Res. Bull., 27, 221-230 (1992).

25. D. C. Golden, J. B. Dixon y C. C. Chen. "Ion exchange, thermal transformations, and oxidizing properties of birnessite". Clays Clay Miner., 34, 511-520 (1986).

26. R. N. DeGuzman, Y. F. Shen, E. J. Neth, S. L. Suib, C. L. O’Young, S. Levine y J. M. Newsam. "Synthesis and characterization of octahedral molecular sieves (OMS-2) having the hollandite structure". Chem. Mater., 6, 815-821 (1994).

27. C. C. Chen, D. C. Golden y J. B. Dixon. "Transformation of synthetic birnessite to cryptomelane. An electron-microscopy study". Clays Clay Miner., 34, 565-571 (1986).

28. O. Prieto y V. Rives. "Preparación y caracterización de óxidos de manganeso no estequiométricos". Bol. Soc. Esp. Ceram. V., 39, 233-238 (2000).

29. JCPDS. Joint Committee on Powder Diffraction Standards, International Centre for Diffraction Data, Pennsylvania, U.S.A. (1977).

30. S. Lowell y J. E. Shields. "Powder Surface Area and Porosity"; Chapman and Hall: London, 1984.

31. V. Rives. "A computer program for analysing nitrogen adsorption isotherms on porous solids". Adsorption Sci. Technol., 8, 95-104 (1991).

32. O. Prieto. Preparación, Caracterización y Evolución Estructural con la Calcinación de Óxidos Mixtos de Manganeso, Tesis Doctoral. Universidad de Salamanca, 2001.

33. O. Prieto, M. del Arco y V. Rives. "Characterisation of K, Na and Li birnessite prepared by oxidation with $\mathrm{H}_{2} \mathrm{O}_{2}$ in a basic medium. Ion exchange properties and study of the calcined products". J. Mater. Sci., 38, 2815-2824 (2002).

34. R. M. Potter y G. R. Rossman. "The tetravalent manganese oxides: identification, hydration, and structural relationships by infrared spectroscopy". Am. Mineral., 64, 1199-1218 (1979).

35. J. P. Parant, R. Olazcuaga, M. Devalette, C. Fouassier y P. Hagenmuller. "Sur quelques nouvelles phases de formule $\mathrm{Na}_{x} \mathrm{MnO}_{2}(x<1)^{\prime \prime}$. J. Solid State Chem. 3, 1-11 (1971).

Recibido: 1.2 .03

Aceptado: 30.11 .03 